\title{
Knowledge visualization with location-based concept mapping in mixed reality
}

\author{
Shengkai Wang ${ }^{\text {a, }} *$, Bing Liu ${ }^{\mathrm{a}}$ \\ ${ }^{a}$ Chair of Cartography, Technical University of Munich, Munich, Germany, shengkai.wang@tum.de, bing.l@tum.de \\ * Corresponding author
}

Keywords: Mixed Reality, knowledge Visualization, Concept Mapping, Spatial Cognition

\begin{abstract}
:
The physical space, which includes the built environment and the corresponding geographical information, can be naturally perceived by human beings by vision. The informational space, which includes cultures, events, social relations and other information that is spatially referenced to objects and locations, can only be represented mentally. The development of information communication technology has promoted the integration of informational space (e.g., humanistic, social-media and cyber spaces) and physical space. This integrated space itself can convey senses of place to people based on what knowledge the places possess. However, it is hard for human to obtain visual experience as what is available in conventional physical space, which leads to a need to research novel theories and methods of the physical-informational space visualization.
\end{abstract}

Mixed reality (MR) offers visual access for humans to perceiving information and acquiring knowledge in real scenes, by augmenting multiple types of virtual objects involves texts, symbols, images, and graphs. With a series of key informative clues such as properties, events, and social relations, individuals are capable to structure knowledge related to certain places, and get contextually specific and episodic understandings of their daily living space. Locationreferenced information is getting increasingly rich in everyday life. Overly rich information can lead to poor attention, high cognitive loads, and complicated interaction with humans, and thus cause inefficient visual searching for key information. Therefore, there is a need to develop an MR-based visualization interface for users to better perceive information and acquire knowledge from real scenes.

Concept maps are classic knowledge visualization used to reduce the amount and complexity of information and to improve user understanding of certain concepts. Node-link map is the basis of concept mapping, which structures knowledge from disorganized information based on informative nodes and links that represent relationships. Concept maps can present an overview and detailed information simultaneously, allowing users to navigate from high-level abstract concepts to low-level information pieces. During the navigation of information, users can discover patterns of knowledge from information series, that is, understanding how certain knowledge is formed and concluded. In addition, with the help of concept maps as an assist of thinking and learning, users are likely to form mental models for recognizing new knowledge patterns, discover new connections of information, and visualize the unknown.

The top-down navigation and learning method based on concept maps is a proper way for individuals to acquire knowledge in real scenes with the help of mixed reality. Instead of searching for useful information from complex visualizations, users can directly navigate between key nodes related to spatial places with sematic information. They can get the main idea that the cartographer or designer would like to convey with the mixed reality knowledge visualization, and figure out why they can come to the point of view by exploring premade knowledge structures. For example, a place can include several feelings that visitors have, such as being clean, crowded, and fashionable. Users who are interested in why there is clean may actively interact with the "clean" node, and discover and explore the sub tree or networks that each node is connected to certain places or concepts like more frequent garbage collection, far with factories, and lower air pollution, and then get the answers of their questions. Some knowledge can be overly informative and complex that consists of lots of information pieces, making it hard to memorize. By exploring the knowledge spatially and semantically simultaneously, like experiencing a story, users can form episodic memory integrated with spatial, temporal, and event information, which makes the memory stronger.

In this project, I would like to solve the following scientific questions: 
a) What are the design principles and strategies for the knowledge visualization with location-based concept mapping in mixed reality;

b) whether the proposed knowledge visualization can improve users' cognitive processes, including knowledge acquiring, memorizing, and using. 